\title{
Análisis morfométrico de los principales tributarios del río Salí en la cuenca de Tapia-Trancas a partir del procesamiento en SIG y sensoramiento remoto
}

\author{
Morphometric analysis of the main Salí river tributaries in the \\ Tapia-Trancas basin applying GIS and remote sensing data
}

\author{
María S. BuSTOS $1,3^{*}$, Sergio M. GEORGIEFF 2,3 \\ 1 Instituto de Ficología, Fundación M. Lillo, Miguel Lillo 251, (T4000JFE) San Miguel de Tucumán, \\ Argentina.solbustos81@gmail.com \\ 2 Facultad de Ciencias Naturales e Instituto Miguel Lillo, Universidad Nacional de Tucumán. Miguel \\ Lillo 205, San Miguel de Tucumán, Tucumán. Argentina.sergio_georgieff@csnat.unt.edu.ar \\ 3 Consejo Nacional de Investigaciones Científicas y Técnicas (CONICET). \\ * Autor al que debe dirigirse la correspondencia: María S. BUSTOS, Instituto de Ficología, Fundación \\ M. Lillo, Miguel Lillo 251, (T4000JFE) San Miguel de Tucumán, Argentina. Mail: msbustos@lillo. \\ org.ar
}

\section{RESUMEN}

El análisis morfométrico provee una descripción de las características de las redes de drenaje de una región y permite anticipar una respuesta frente a los fenómenos climáticos. En este trabajo se presentan los resultados del análisis morfométrico realizado sobre los principales tributarios del río Salí en la cuenca de Tapia-Trancas, Tucumán, Argentina. Los parámetros morfométricos fueron calculados en base a la metodología de Horton-Strahler con apoyo de herramientas de sistemas de información geográfica y datos de sensoramiento remoto (modelo de elevación digital SRTM). La relación entre orden de los ríos, longitud media y el número de ríos, sigue tendencias geométricas. El coeficiente de almacenamiento indica que en los ríos Choromoro, Tapia y Acequiones (valores altos de Rho) se dan mayores tiempos para alcanzar los caudales de punta y los procesos erosivos están atenuados. Los valores más altos del coeficiente de torrencialidad caracterizaron a las cuencas de los ríos Candelaria, Tala y arroyo India Muerta, indicando mayor ocurrencia de flujos

Ref. bibliográfica: Bustos, M. S.; Georgieff, S. M. 2020. "Análisis morfométrico de los principales tributarios del río Salí en la cuenca de Tapia-Trancas a partir del procesamiento en SIG y sensoramiento remoto". Acta geológica lilloana 32 (1-2): 27-46. Fundación Miguel Lillo, Tucumán, Argentina. D.O.I.: https://doi. org/10.30550/j.agl/2020.32.1-2/2

- Recibido: 7 de febrero 2020 - Aceptado: 2 de setiembre 2020.

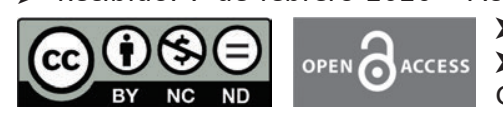

> URL de la revista: http://actageologica.lillo.org.ar

- Esta obra está bajo una Licencia Creative Commons Atribución - No Comercial - Sin Obra Derivada 4.0 Internacional. 
torrenciales e inundaciones aluvionales veloces. La extensión media del escurrimiento fue mayor en los ríos Tapia y Choromoro lo cual implica un mayor tiempo para alcanzar los caudales de punta. El río Acequiones fue el de mayor densidad de drenaje indicando que la red hidrológica se desarrolla sobre rocas más impermeables. La frecuencia de ríos (Fr) muestra un escaso desarrollo en la subcuenca del arroyo India Muerta, que se condice con su baja densidad de drenaje y escasa superficie. Las subcuencas de los ríos Tapia, Tala y Candelaria, tienen mayores valores de Fr lo cual representa una mayor disponibilidad de agua en superficie, menor permeabilidad de los terrenos y mayor susceptibilidad a la erosión. El análisis morfométrico en base al procesamiento de datos de sensores remotos permite caracterizar las redes de drenaje de los tributarios del río Salí en la cuenca de Tapia-Trancas e identificar las subcuencas que presentan una mayor susceptibilidad a la erosión.

Palabras clave - Parámetros morfométricos, Tapia-Trancas, río Salí, SIG.

\begin{abstract}
"Morphometric analysis of the main Salí river tributaries in the Tapia-Trancas basin using GIS and remote sensing data".- This paper presents the results of the morphometric analysis performed on the main Sali tributaries at the Tapia-Trancas basin, Tucumán, Argentina. The morphometric parameters were computed according to Horton-Strahler methodology with the support of geographic information systems and remote sensing data (SRTM digital elevation model). The relationship between the order of the rivers, average length and the number of rivers, follows geometric tendencies. The storage coefficient indicates longer times to reach peak flows and attenuated erosion processes for the Choromoro, Tapia and Acequiones rivers (high values of Rho). Higher values of the torrentiality coefficient characterized the watersheds of Candelaria river, Tala river and India Muerta Creek, indicating the occurrence of flash floods and fast alluvial floods. The average extent of runoff was higher in the Tapia and Choromoro rivers, which would imply a longer time to reach peak flow. The Acequiones River had the highest drainage density, indicating the development of the hydrological network on impermeable rocks. The frequency of rivers was indicative of scarce development in the case of the India Muerta stream, which is also proven by its low drainage density and scarce surface area. The sub-basins of the Tapia, Tala, and Candelaria rivers had higher values of Fr, which represented a greater availability of surface water, less permeability and greater susceptibility to erosion. The morphometric analysis based on the processing of remote sensing data allowed the characterization of the drainage networks of the main tributaries of the Sali river in the region of Tapia-Trancas basin and the identification of those which present a greater susceptibility to erosion.
\end{abstract}

Keywords - Morphometric parameters, Tapia-Trancas, Salí river, GIS. 


\section{INTRODUCCIÓN}

Una cuenca hidrográfica y su red de drenaje pueden ser descriptas en función de parámetros morfométricos. El análisis morfométrico, desarrollado por Horton (1945) y Strahler (1957), provee una descripción cuantitativa de las redes de drenaje (Pande y Moharir 2017). Además, permite comprender el comportamiento de las cuencas y las redes de drenaje frente a los efectos de diversos controles como el clima, la geología, la geomorfología y la estructura (Knighton 1998; Gordon et al. 2004; Gaspari et al. 2012; Biswas et al. 2014). Por otra parte, el estudio morfométrico de las cuencas ha probado ser una herramienta útil para el diagnóstico, la conservación y la gestión de los recursos naturales (Kulkarni 2013; Kumar 2013) al brindar conocimiento y datos para la toma de decisiones técnico-políticas, planificación estratégica y la delineación de zonas de manejo prioritario del riesgo (Nageswara et al. 2010; Biswas et al. 2014).

Esta metodología ha sido aplicada en cuencas de Argentina, con diferentes objetivos. La influencia de distintos controles geológicos: litología, estructura y tectónica en el desarrollo y organización de las redes fue abordada por Salomón y Soria (2002), Doffo y González Bonorino (2005), Mesa (2006) y Beltramone (2007) en cuencas de las provincias de Mendoza, Tucumán y Córdoba. También, Gil et al. (2009) trabajaron en el sector Sur del Sistema de Ventania, provincia de Buenos Aires, reconociendo su utilidad para conocer la respuesta ante eventos torrenciales, en áreas que no cuentan con información meteorológica e hidrométrica. Genchi et al. (2011) combinaron el análisis morfométrico con el número de curva, en cuencas del Golfo de San Matías, realizando una descripción física cuantitativa y explicando los efectos hidrológicos bajo diferentes usos del suelo. Gil (2011) determinó la dinámica torrencial y la influencia de la infiltración y el escurrimiento, en la cuenca del arroyo El Negro (Buenos Aires) teniendo en cuenta los índices morfométricos, la confección de un mapa hidrogeomorfológico y el análisis de registros de precipitación y caudales. Por su parte, Esper Angillieri y Perucca (2014) destacaron el aporte de la morfometría para la zonificación de la susceptibilidad y peligrosidad ante la ocurrencia de eventos en la cuenca del río Seco (San Juan).

Los sistemas de información geográfica (SIG) constituyen una herramienta de apoyo para estudiar el comportamiento hidrológico de las cuencas. El avance en la tecnología de los SIG y los modelos de elevación digital (DEM) permiten dilucidar las variables que actúan a una misma escala espacial y altitudinal sobre la red de drenaje (Gaspari et al. 2012). Además, brinda una forma precisa, rápida y de bajo costo para el estudio de los sistemas hidrológicos (Rai et al. 2017). Al respecto, Navarro y Cano (2017) resaltaron la importancia de trabajar sobre la base de datos satelitales registrados para un mismo tiempo y bajo las mismas condiciones como una forma de dar mayor confiabilidad a los índices obtenidos. La combinación del análisis morfométrico apoyado con SIG y el trabajo de campo en áreas diagnóstico, es útil además para comparar y transferir resultados a cuencas cercanas con características similares (Gil et al. 2009). En áreas donde no existe adecuada información climática de base, el SIG y el análisis morfométrico permiten obtener información para organizar el uso de los recursos hídricos, así como, analizar la susceptibilidad y/o peligrosidad frente 
a la ocurrencia de flujos destructivos (Angillieri et al. 2016). En Tucumán, Busnelli y Horta (2014) aplicaron SIG para integrar cálculos morfométricos y análisis multitemporales de cambios de sinuosidad, destacando la utilidad de la metodología para determinar el comportamiento hidrológico, el grado de torrencialidad y la respuesta ante eventos lluviosos extraordinarios (vinculados a inundaciones), en ríos donde no existen registros de caudales. Si bien, el análisis morfométrico constituye una base, es importante contar con estaciones de medición con una escala temporal adecuada acorde a las características de cada área (Genchi et al. 2011).

La combinación de morfometría con información de otras disciplinas facilita, además, una mejor caracterización de las cuencas hídricas (Díaz et al. 2015). Esto resulta clave para la planificación y manejo de las cuencas, la gestión de emergencias ambientales y el ordenamiento ambiental, favoreciendo la organización de las comunidades y su entorno (Delgado y Gaspari 2010; Gaspari et al. 2012; Díaz Gómez et al. 2017).

La cuenca semiárida de Tapia-Trancas constituye una región representativa del Noroeste Argentino (Figura 1), caracterizada por la ocurrencia de procesos degradatorios que vulneran la provisión adecuada del recurso hídrico, así como la conservación de los suelos en la zona (Bustos y Georgieff 2016). El embalse Celestino Gelsi fue construido en la salida de la cuenca Tapia-Trancas para provisión de agua (potable, para riego y uso industrial), atenuación de crecidas, generación de energía eléctrica y recreación (Adler 2004). Desde la puesta en funcionamiento del embalse en 1966, los estudios batimétricos llevados a cabo (Pacha 2005; Huespe et al. 2009) revelaron la continua y acentuada depositación de sedimentos que produjo una disminución de más del $30 \%$ del volumen original del embalse con consecuencias en el funcionamiento en la obra (Adler 2004; Díaz 2006). Esto implicó que a raíz de la acumulación de sedimentos frente a las torres de toma de agua se debió implementar un sistema de captación alternativo para evitar el arrastre de sedimentos y de aguas con bajos tenores de oxígeno disuelto o altos valores de sales según lo explica Adler (2004). El objetivo general de este trabajo es determinar y comparar las características morfométricas de las cuencas para identificar los factores naturales que controlan la producción de caudal y sedimento en la cuenca. Por ello, se propusieron los siguientes objetivos específicos: i) caracterizar la red hidrológica de las cuencas de aporte de los principales tributarios del Salí en la cuenca de Tapia-Trancas en base a parámetros morfométricos; ii) identificar los factores que controlan los procesos erosivos en las subcuencas tributarias del Salí y iii) reconocer las diferencias en el comportamiento hidrológico de los tributarios del Salí en base a los resultados obtenidos.

\section{ÁREA DE ESTUDIO}

La cuenca de Tapia-Trancas es un valle semiárido ubicado en el centro-norte de la provincia de Tucumán y sur de Salta (figura 1). La red hidrográfica principal, tiene por eje al río Salí que nace al norte con el nombre de Tala y recibe por el margen oriental al río Candelaria. Por el margen occidental los tributarios son los ríos Acequiones, Choromoro, Vipos, Tapia y arroyo India Muerta. El valle es una depresión 
tectónica resultante de la compresión cenozoica, durante la orogénesis andina (Abascal 2005). El límite occidental está configurado por las Cumbres Calchaquíes. En el sector oriental se encuentran las sierras de Medina y de Candelaria. En el sector sur, la aproximación de las sierras de Medina y de San Javier forman un cierre estructural donde se ubica el Embalse Celestino Gelsi.

Tres unidades litoestratigráficas conforman la cuenca: 1) el basamento formado por granitoides y rocas metasedimentarias de grados bajo y mediano (Proterozoico-Paleozoico inferior; Formaciones Medina y Puncoviscana), 2) los depósitos del Grupo Salta, de espesor reducido en el área (Cretácico-Paleógeno), y 3) depósitos que corresponden al desarrollo de cuencas de antepaís e intramontanas (Grupo Choromoro y Formaciones Acequiones y Ticucho; Neógeno-reciente) (Abascal, 2005). Las rocas del basamento metamórfico afloran en las Cumbres Calchaquíes y en la sierra de Medina y sobre estas se encuentra discordante una potente sucesión de estratos continentales del Grupo Salta (Abascal, 2005). A su vez, en discordancia sobre el Grupo Salta existen depósitos lacustres y aluviales cenozoicos, deformados por la orogénesis andina (Abascal, 2005).

El clima es de tipo semiárido, BSk según la clasificación de Koppen con un gradiente de precipitación entre $500-900 \mathrm{~mm}$ anuales y una temperatura media anual entre los $12^{\circ}$ y $18^{\circ} \mathrm{C}$ (Mendoza y González 2011). La vegetación de la cuenca corresponde a la ecoregión del Chaco Serrano, ubicado en un sector de transición con las

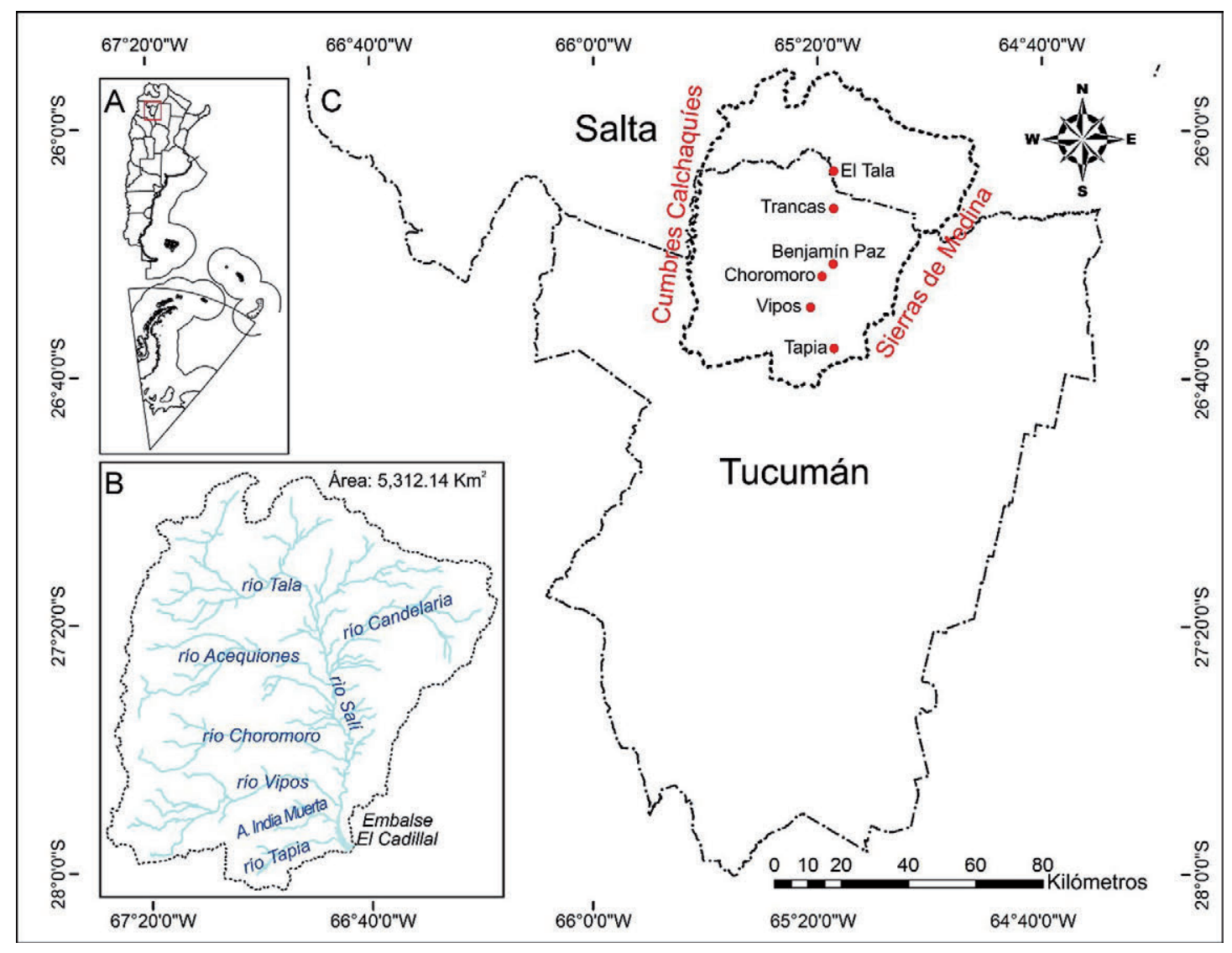

Figura 1. Ubicación de la Cuenca de Tapia-Trancas, provincias de Salta y Tucumán. 
ecoregiones de Yungas y Chaco Semiárido (Torrela y Adámoli 2005; Mendoza y González 2011).

\section{MATERIALES Y MÉTODOS}

La confección de los mapas hidrológicos y la obtención de parámetros morfométricos se realizaron a partir del modelo de elevación digital (DEM) SRTM de la National Geospatial-Intelligence Agency de la NASA, de $90 \mathrm{~m}$ (Farr et al. 2007). Este fue remuestreado a un tamaño de pixel de $30 \mathrm{~m}$ y se realizó un corregistro imagen a imagen seleccionando puntos de control a partir de la imagen base Landsat 5TM (path 230/ row 078 del mes de julio de 2007). Luego fue proyectado al Sistema Posgar 94, Zona 3, el error resultante del proceso de georeferenciacion por puntos fue menor de $30 \mathrm{~m}$. Posteriormente el DEM fue procesado aplicando la metodología de Fleming y Dohan (2009) con el software HEC-GEO HMS. El pre-procesamiento realizado consistió en el relleno de depresiones erróneas. En base al DEM corregido se generaron grillas de dirección y acumulación de flujo a partir de las cuales se definió la red de drenaje estableciendo un umbral de acumulación de flujo de $16,26 \mathrm{~km}^{2}$, equivalente al 0,3\% del área. Luego, de forma automática, se delineó la red de drenaje y se delimitaron subcuencas y microcuencas mediante la identificación manual de puntos de salida que facilitan la detección del área contribuyente aguas arriba. Posteriormente se realizaron operaciones espaciales para simplificar la información resultante del procesamiento automático, de forma que represente adecuadamente la realidad del terreno. Con la base de información espacial generada se obtuvieron los datos cuantitativos necesarios para calcular los parámetros morfométricos (Tabla 1) de acuerdo al Método de Horton-Strahler (Horton 1945; Strahler 1957). Este se basa en tres principios básicos: 1) los tributarios que se originan en las nacientes se designan con el orden 1,2) la unión de dos tributarios de orden u forma un segmento de canal de orden $\mathrm{u}+1$ y 3) la unión de dos ríos de distinto orden u y v, donde $\mathrm{v}>$ $\mathrm{u}$, forma un segmento aguas abajo de orden igual al del segmento de orden mayor (v) (Knighton 1998).

Además, la caracterización morfométrica de los tributarios del río Salí, se complementó con el análisis de los perfiles longitudinales y se cuantificó la proporción de áreas ocupadas por diferentes litologías en cada tributario. Esto último se hizo en base a la litología descripta en la hoja Geológica 2765 - II San Miguel de Tucumán (González et al. 2000). La hoja geológica en formato analógico (papel) fue digitalizada, georeferenciada por puntos (error menor de $30 \mathrm{~m}$ ) y proyectada al Sistema Posgar 94, Zona 3. Luego se digitalizaron las principales unidades litológicas representadas en ella y se realizó un análisis estadístico por zona para calcular la proporción de cada tipo litológico en cada subcuenca. Estos datos permitieron identificar diferencias locales debidas a la litología, la estructura o a cambios del material del lecho.

Los suelos y sus propiedades hidrológicas están íntimamente asociados a la litología de la cual derivan (Renschler et al., 1999). En la cuenca del río Tipas, uno de los tributarios del rio Acequiones en Tapia-Trancas, Guido (1987) reconoció que la distribución de la erodabilidad de los suelos se halla en relación con la litología y 
Tabla 1. Parámetros morfométricos: nombres y ecuaciones.

\begin{tabular}{|c|c|c|}
\hline Parámetro & Ecuación & Descripción \\
\hline Relación de bifurcación (Rb) & $\mathrm{Rb}=\mathrm{Nu} / \mathrm{N}(\mathrm{u}+1)$ & $\begin{array}{l}\text { Nu: número de ríos de un } \\
\text { orden dado } \\
\text { Nu+1: número de ríos del } \\
\text { orden inmediato superior. }\end{array}$ \\
\hline Relación de longitud (RI) & $\mathrm{RI}=\mathrm{Lu} / \mathrm{L}(\mathrm{u}-1)$ & $\begin{array}{l}\text { Lu: longitud media de los ríos } \\
\text { de un orden dado } \\
\text { Lu-1: longitud media de los } \\
\text { ríos de un orden inferior. }\end{array}$ \\
\hline Densidad de Drenaje (Dd) & $\mathrm{Dd}=\mathrm{Lt} / \mathrm{A}$ & $\begin{array}{l}\text { Lt: longitud total de los ríos } \\
\text { de una cuenca dada } \\
\text { A: área de la cuenca. }\end{array}$ \\
\hline Frecuencia de ríos (Fr) & $\mathrm{Fr}=\mathrm{Nt} / \mathrm{A}$ & $\begin{array}{l}\mathrm{Nt} \text { : número total de ríos } \\
\mathrm{A} \text { : área de la cuenca }\end{array}$ \\
\hline $\begin{array}{l}\text { Coeficiente de almacenamiento } \\
(\mathrm{RHO})\end{array}$ & $\mathrm{RHO}=\mathrm{R} \mid$ media/Rb media & $\begin{array}{l}\mathrm{RI} \text { media: relación de } \\
\text { longitud media } \\
\mathrm{Rb} \text { media: relación de } \\
\text { bifurcación media de la red } \\
\text { drenaje. }\end{array}$ \\
\hline Coeficiente de torrencialidad (Ct) & $\mathrm{Ct}=\mathrm{N} 1 / \mathrm{A}$ & $\begin{array}{l}\text { N1: número de ríos de primer } \\
\text { orden } \\
\text { A: área de la cuenca }\end{array}$ \\
\hline $\begin{array}{l}\text { Extensión media del escurrimiento } \\
\text { (E) }\end{array}$ & $E=A / 4 x L t$ & $\begin{array}{l}\text { A: es el área de la cuenca. } \\
\text { Lt: es la longitud total de los } \\
\text { ríos de la cuenca. }\end{array}$ \\
\hline
\end{tabular}

el mesoclima. Siguiendo esta propuesta, las unidades litológicas del área se agruparon considerando la susceptibilidad a la erosión: 1) los mayores valores se asignaron a sedimentos y sedimentitas cuaternarios de la cuenca baja a media, 2) los valores intermedios corresponden a sedimentitas del Neógeno y, 3) los valores mínimos de erodabilidad son asignados a la cuenca alta formada por basamento cristalino y sedimentitas cretácicas (Bustos, 2014).

\section{RESULTADOS}

\section{Área}

La cuenca de Tapia-Trancas comprende una superficie de 5.312,14 $\mathrm{km}^{2}$, de la cual en porcentaje y en orden de importancia corresponden: $33,63 \%$ a la subcuenca del río Tala, $16,27 \%$ a la subcuenca del río Choromoro, $13,62 \%$ a la subcuenca del río Vipos, $11,4 \%$ a la subcuena del río Candelaria, $10,75 \%$ a la subcuenca del río Acequiones, el 6,38\% a las subcuencas del río Tapia y arroyo India Muerta y el $8 \%$ restante a los arroyos de menor envergadura y caudal intermitente que nacen en las vertientes orientales y occidentales de la cuenca. La figura 2 muestra la red hídrica de la cuenca y las subcuencas de los principales tributarios del río Salí. 


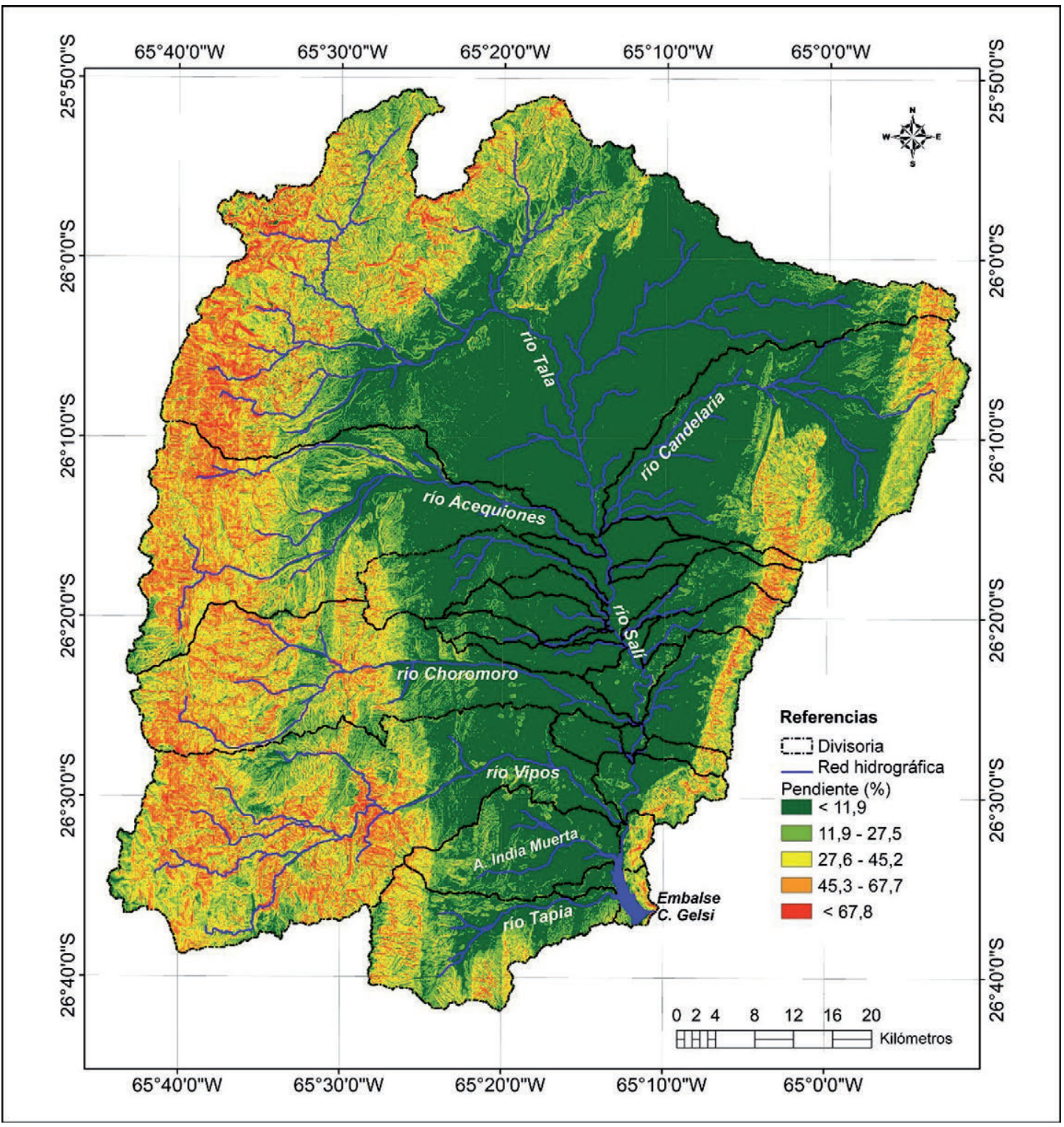

Figura 2. Mapa de pendientes (\%) de la Cuenca Tapia-Trancas con indicación de las principales subcuencas y red de drenaje.

\section{Longitud total}

La red de drenaje de mayor longitud corresponde al río Tala con casi $400 \mathrm{~km}$, las demás subcuencas presentaron valores inferiores a $200 \mathrm{~km}$ y la de menor longitud fue la subcuenca del Arroyo India Muerta con menos de $50 \mathrm{~km}$.

\section{Leyes de Composición de las redes de drenaje}

Los datos de orden de los ríos, longitud media del orden, relación de bifurcación y relación de longitud se muestran en la tabla 2. 
Tabla 2. Parámetros morfométricos de las redes de drenaje de los tributarios del río Salí en la cuenca de Tapia-Trancas. Orden de los ríos, longitud media del orden, relación de bifurcación, relación de longitud de las principales subcuencas.

\begin{tabular}{|c|c|c|c|c|c|}
\hline $\begin{array}{l}\text { Número de } \\
\text { orden }\end{array}$ & $\begin{array}{l}\text { Número de } \\
\text { corrientes } \\
\text { del orden } \\
(\mathrm{Nu})\end{array}$ & $\begin{array}{l}\text { Long. total del } \\
\text { orden }(\mathrm{km})\end{array}$ & $\begin{array}{l}\text { Long. media del } \\
\text { orden }(\mathbf{k m})\end{array}$ & $\begin{array}{l}\text { Relación de } \\
\text { bifurcación } \\
\text { (Rb) }\end{array}$ & $\begin{array}{l}\text { Relación } \\
\text { de longitud } \\
\text { (RI) }\end{array}$ \\
\hline \multicolumn{6}{|l|}{ río Tapia } \\
\hline 2 & 2 & 7,69 & 3,84 & 2,00 & 1,27 \\
\hline 3 & 1 & 16,14 & 16,14 & & 4,20 \\
\hline Promedio & & & & 2,00 & 2,74 \\
\hline \multicolumn{6}{|l|}{ río Vipos } \\
\hline 1 & 11 & 74,20 & 6,75 & 2,20 & \\
\hline 2 & 5 & 39,61 & 7,92 & 2,50 & 0,53 \\
\hline 3 & 2 & 40,23 & 20,11 & & 1,02 \\
\hline Promedio & & & & 2,35 & 0,77 \\
\hline \multicolumn{6}{|c|}{ arroyo India Muerta } \\
\hline 1 & 3 & 20,79 & 6,93 & 3,00 & \\
\hline 2 & 1 & 7,29 & 7,29 & & 1,05 \\
\hline \multicolumn{6}{|l|}{ río Choromoro } \\
\hline 1 & 8 & 51,30 & 6,41 & 2,00 & \\
\hline 2 & 4 & 12,95 & 3,24 & 4,00 & 0,50 \\
\hline 3 & 1 & 34,61 & 34,61 & & 10,69 \\
\hline Promedio & & & & 3,00 & 5,60 \\
\hline \multicolumn{6}{|c|}{ río Acequiones } \\
\hline 1 & 9 & 50,18 & 5,58 & 2,25 & \\
\hline 2 & 4 & 56,77 & 14,19 & 2,00 & 2,55 \\
\hline 3 & 2 & 27,84 & 13,92 & & 0,98 \\
\hline Promedio & & & & 2,13 & 1,76 \\
\hline \multicolumn{6}{|l|}{ río Tala } \\
\hline 1 & 40 & 205,70 & 5,14 & 3,64 & \\
\hline 2 & 11 & 110,15 & 10,01 & 2,75 & 0,54 \\
\hline 3 & 4 & 32,57 & 8,14 & 2,00 & 0,30 \\
\hline 4 & 2 & 40,85 & 20,42 & & 1,25 \\
\hline Promedio & & & & 2,80 & 0,70 \\
\hline \multicolumn{6}{|l|}{ río Candelaria } \\
\hline 1 & 13 & 95,31 & 7,33 & 4,33 & \\
\hline 2 & 3 & 18,10 & 6,03 & 3,00 & 0,19 \\
\hline 3 & 1 & 29,16 & 29,16 & & 1,61 \\
\hline Promedio & & & & 3,67 & 0,9 \\
\hline
\end{tabular}

De acuerdo a Singh et al. (2014), la obtención automática de las redes de drenaje es una metodología precisa, rápida y actualizada que permite el análisis regional de extensas redes de drenaje como la que se presenta en este trabajo. De esta manera, los resultados para la relación de bifurcación media de las cuencas analizadas tuvieron valores entre 2 (río Tapia) y 3,67 (río Candelaria), mientras que la Relación de longitud media varía entre 0,7 (menor valor) en río Tala y 5,60 en río Choromoro. Las redes de drenaje estudiadas cumplen la progresión geométrica tanto inversa (número de ríos vs orden) como directa (longitud media del orden vs orden) de acuerdo a las leyes de composición de las redes de drenaje (figura 3).

Los valores de los coeficientes de almacenamiento (RHO), de torrencialidad $(\mathrm{Ct})$, extensión media del escurrimiento superficial $(\mathrm{E})$, densidad de drenaje $(\mathrm{Dd})$ 


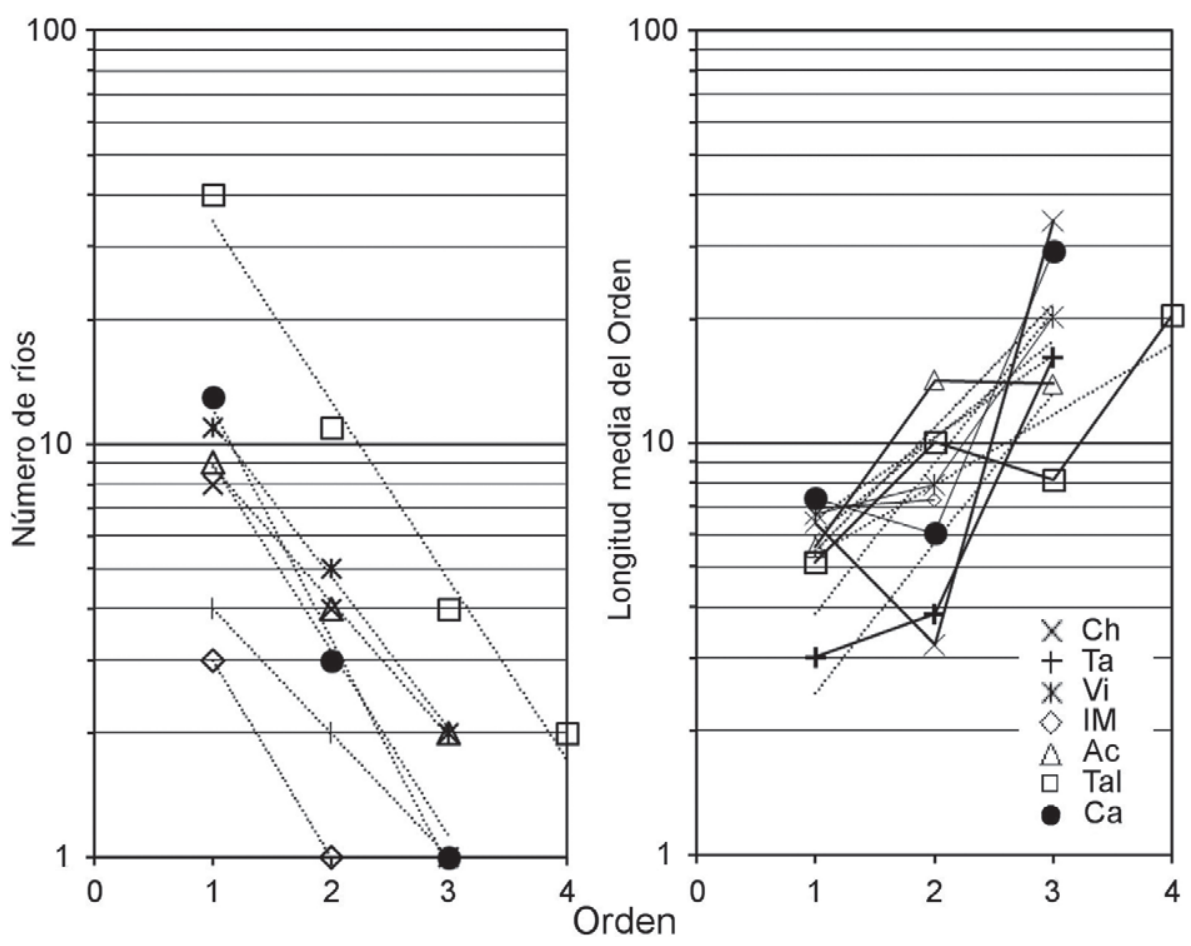

Figura 3. Leyes de composición de las redes de drenaje: representación en escala logarítmica: a) número de ríos en función del orden, b) la longitud media en función del orden de los ríos. Referencias: Ta (Tapia), IM (India Muerta), Vi (Vipos), Ch (Choromoro), Ac (Acequiones), Ca (Candelaria), Tal (Tala).

y frecuencia de ríos (Fr) de las cuencas tributarias del río Salí se presentan en la figura 4. El mayor valor del coeficiente de almacenamiento se obtuvo para la red de drenaje del río Choromoro $(1,866)$ y el menor valor para el río Candelaria $(0,246)$. La densidad de drenaje fue máxima en el río Acequiones $(0,237)$, seguida del río Candelaria $(0,235)$ y finalmente el río Tala $(0,218)$. El mínimo valor de Densidad de drenaje (Dd) fue obtenido para el arroyo India Muerta con 0,033. En cuanto al índice de extensión media del escurrimiento superficial, el río Choromoro presentó el valor más alto $(2,186)$ mientras que valor mínimo calculado fue para India Muerta $(0,001)$. El máximo valor del coeficiente de torrencialidad correspondió al Arroyo India Muerta $(0,024)$ mientras que el río Choromoro tuvo el menor coeficiente $(0,009)$. La frecuencia de ríos tuvo un valor máximo en el río Tapia $(0,033)$ mientras que el menor valor $(0,001)$ se obtuvo en el arroyo India Muerta.

\section{Perfiles longitudinales}

Los perfiles longitudinales de los ríos principales se muestran en la figura 5. En general las formas de los perfiles son cóncavas, mostrando las pendientes más pronunciadas en los sectores aguas arriba y disminuyendo de forma gradual hacia las zonas bajas del valle Tapia - Trancas hasta la confluencia con el río Salí. Los ríos Tapia, Candelaria y arroyo India Muerta muestran perfiles con una pendiente más 

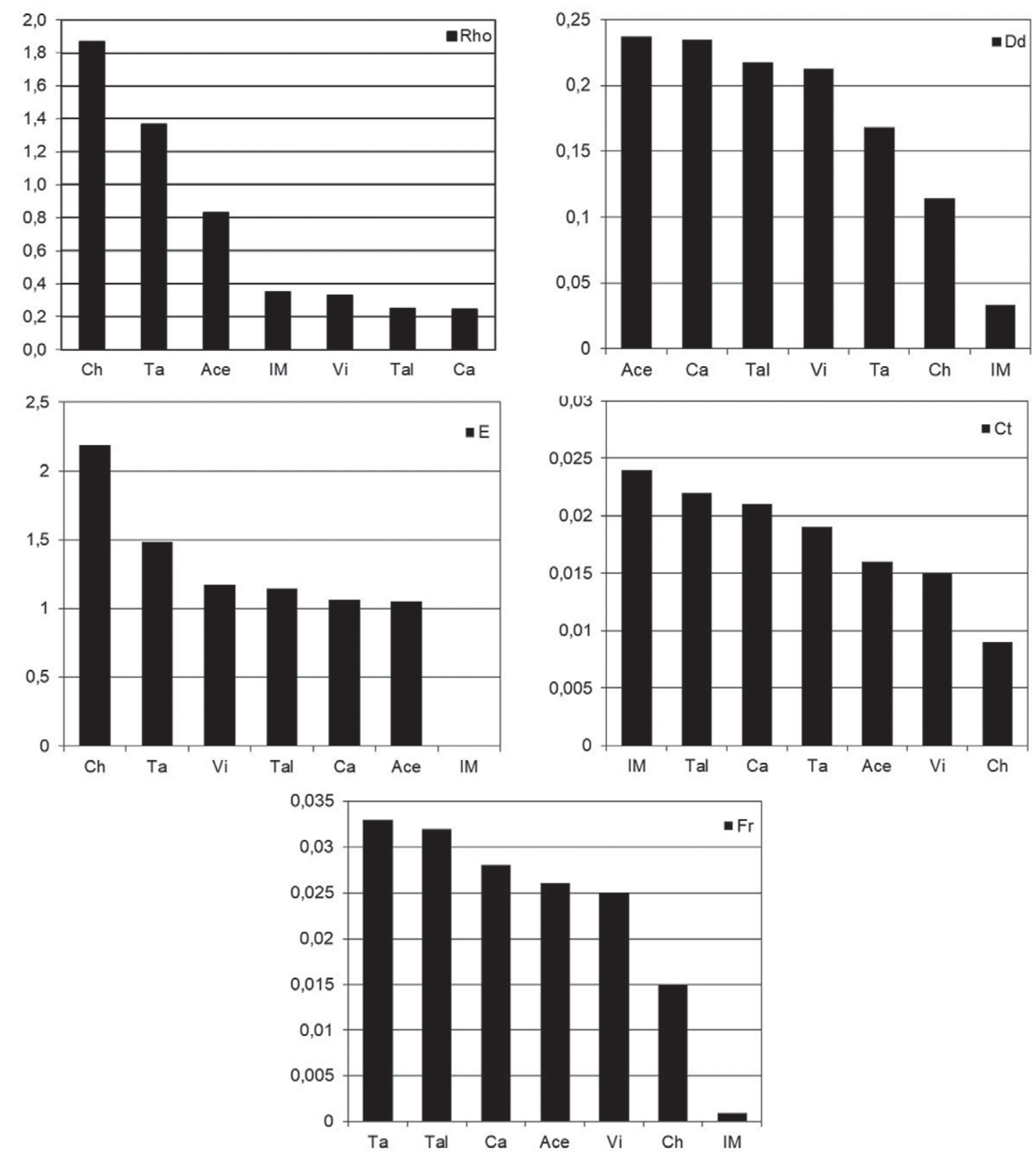

Figura 4. Parámetros morfométricos calculados para las distintas subcuencas: Rho (coeficiente de almacenamiento), Dd (densidad de drenaje), E (extensión media del escurrimiento superficial), Ct (coeficiente de torrencialidad) y Fr (frecuencia de ríos), de las redes de drenaje de los principales tributarios del Salí en el área de estudio. Referencias: Ace (Acequiones), Ca (Candelaria), Ch (Choromoro), IM (India Muerta), Tal (Tala), Ta (Tapia), Vi (Vipos).

uniforme a lo largo de todo el cauce desde las nacientes hasta la desembocadura. Estos cursos son además los de menor longitud. Los perfiles de los ríos Tala, Choromoro y Vipos presentan quiebres de la pendiente (knickpoints), posiblemente asociados a estructuras geológicas (fallas o pliegues en subsuelo, ver González et al. 2000; Abascal, 2005; Córdoba, 2013).

Las figuras 6 y 7 muestran la distribución espacial y la proporción de las diferentes litologías en el área de drenaje de los tributarios estudiados. En las cuencas de Vipos, Choromoro, Acequiones y Tapia predomina el basamento metamórfico. 


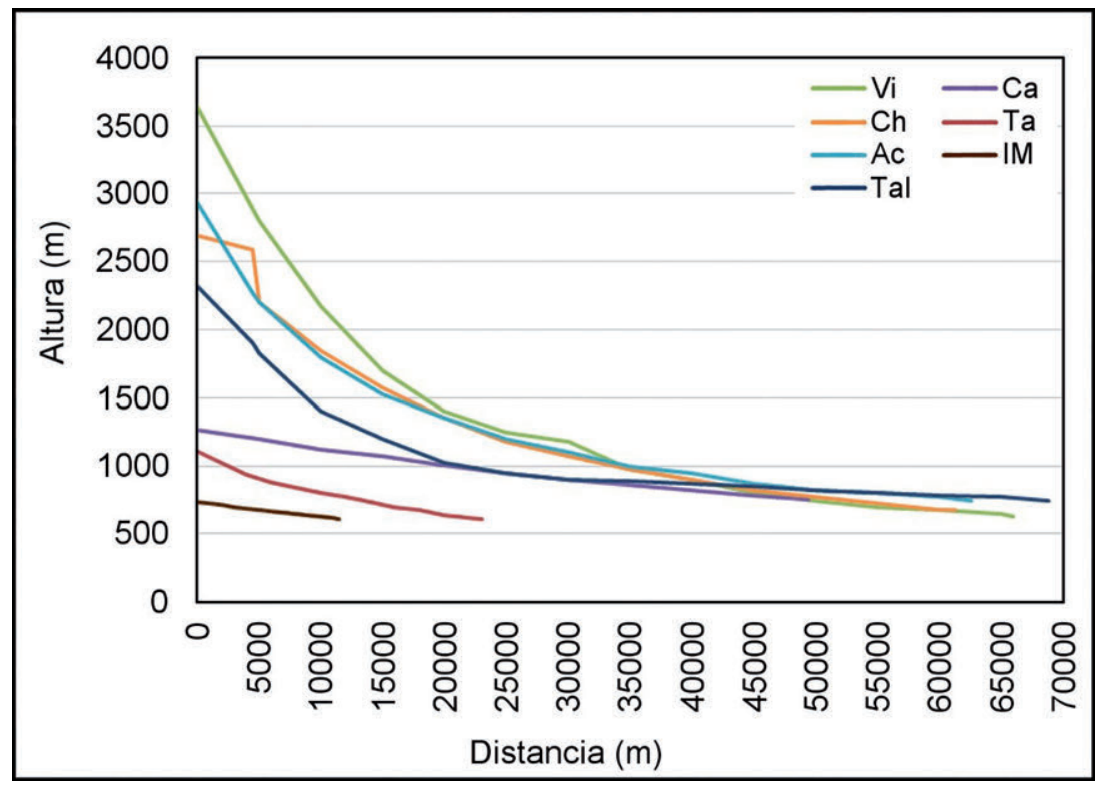

Figura 5. Perfiles longitudinales de los principales tributarios del Salí en la Cuenca de Tapia-Trancas. Referencias: Ac (Acequiones), Ca (Candelaria), Ch (Choromoro), IM (India Muerta), Ta (Tapia), Tal (Tala), y Vi (Vipos).

Mientras en las cuencas del Tala, Candelaria e India Muerta prevalecen los materiales Terciarios y Cuaternarios (conglomerados, arenas y limos).

\section{DISCUSIÓN Y CONCLUSIONES}

El análisis morfométrico a partir de datos derivados de teledetección y procesamiento en SIG permite la caracterización de las redes de drenaje de los tributarios del Salí en la cuenca de Tapia-Trancas. Los datos obtenidos constituyen la base para identificar los factores que controlan la génesis y el comportamiento hidrológico de los tributarios e identificar áreas prioritarias de manejo. Los valores absolutos obtenidos para cada coeficiente son indicativos de los procesos y pueden estar subestimando algunas características particulares a escala de cuenca; sin embargo, la aplicación de criterios y metodologías comunes permiten realizar comparaciones entre las cuencas y determinar un ordenamiento escalado de los procesos y características principales.

Las cuencas analizadas mostraron tendencias geométricas al relacionar el orden de los ríos, con la longitud media y el número de ríos, como se espera de acuerdo a las leyes de composición de las redes de drenaje (Knighton 1998; Gordon et al. 2004; Doffo y González Bonorino 2005; Busnelli 2009). La relación de longitud (Rl) manifiesta la proporción de incremento en la longitud de los ríos a medida que aumenta el número de orden. Así, en el río Tapia la longitud de los ríos de órdenes superiores casi triplicó a las de los órdenes inferiores. En la cuenca del río Acequiones la relación fue del doble, mientras en el río Choromoro, los órdenes superiores casi sextuplicaron a los inferiores en longitud. Los cambios en $\mathrm{Rl}$ de un orden a otro indican su etapa juvenil tardía de desarrollo geomorfológico de acuerdo a Pande y Moharir 


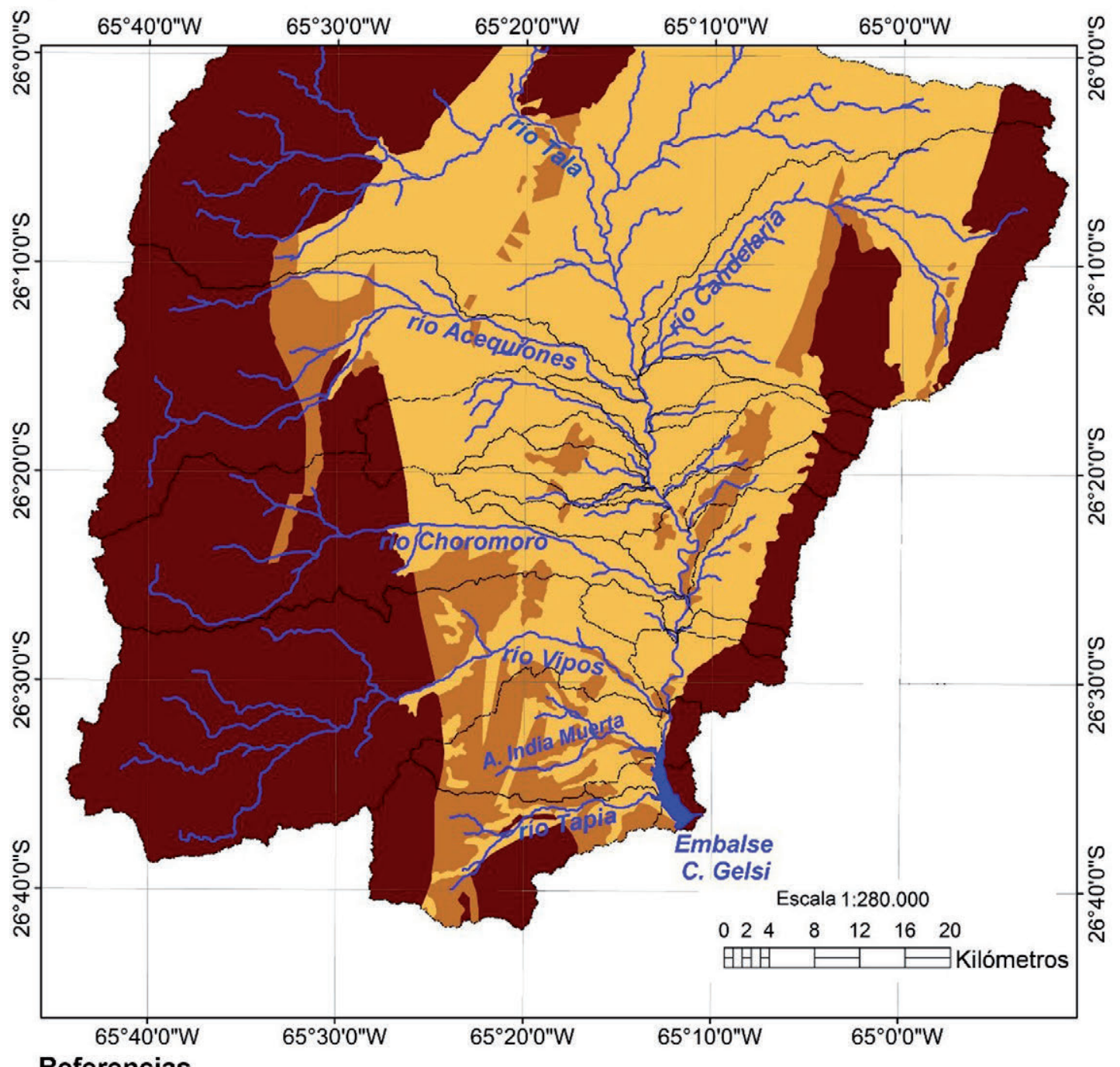

Referencias

Susceptibilidad a la erosión

$\square$ 0,05-0,06 (sedimentos actuales y sedimentitas cuaternarias)

0,04 (sedimentitas neógenas)

0,02 (basamento cristalino y sedimentitas cretácicas)

Figura 6. Mapa de susceptibilidad a la erosión para la cuenca de Tapia-Trancas. Las unidades fueron agrupadas de acuerdo a las litologías propuestas por González et al. (2000). El criterio de agrupamiento es similar al propuesto por Guido (1987) y discutido en el texto (ver Materiales y Métodos). Los límites de las unidades tienen un control tectónico dado por lineamientos, fracturas, fallas y pliegues (según los criterios de Abascal, 2005; Córdoba, 2013; Georgieff et al. 2014).

(2017). Campo et al. (2012) indican que valores de relación de longitud mayores a 3 representan cuencas donde hay una concentración paulatina de caudales a lo largo del colector principal. En este estudio únicamente el río Choromoro tiene un valor alto de $\mathrm{Rl}>3$ mientras que los restantes tributarios tienen valores de $\mathrm{Rl}<3$ por lo que correspondería clasificarlas como cuencas torrenciales (Campo et al. 2012).

En las redes de drenaje estudiadas, la relación de bifurcación media $(\mathrm{Rb})$ muestra valores en torno a 2 , considerados bajos, característicos de cuencas de menor 


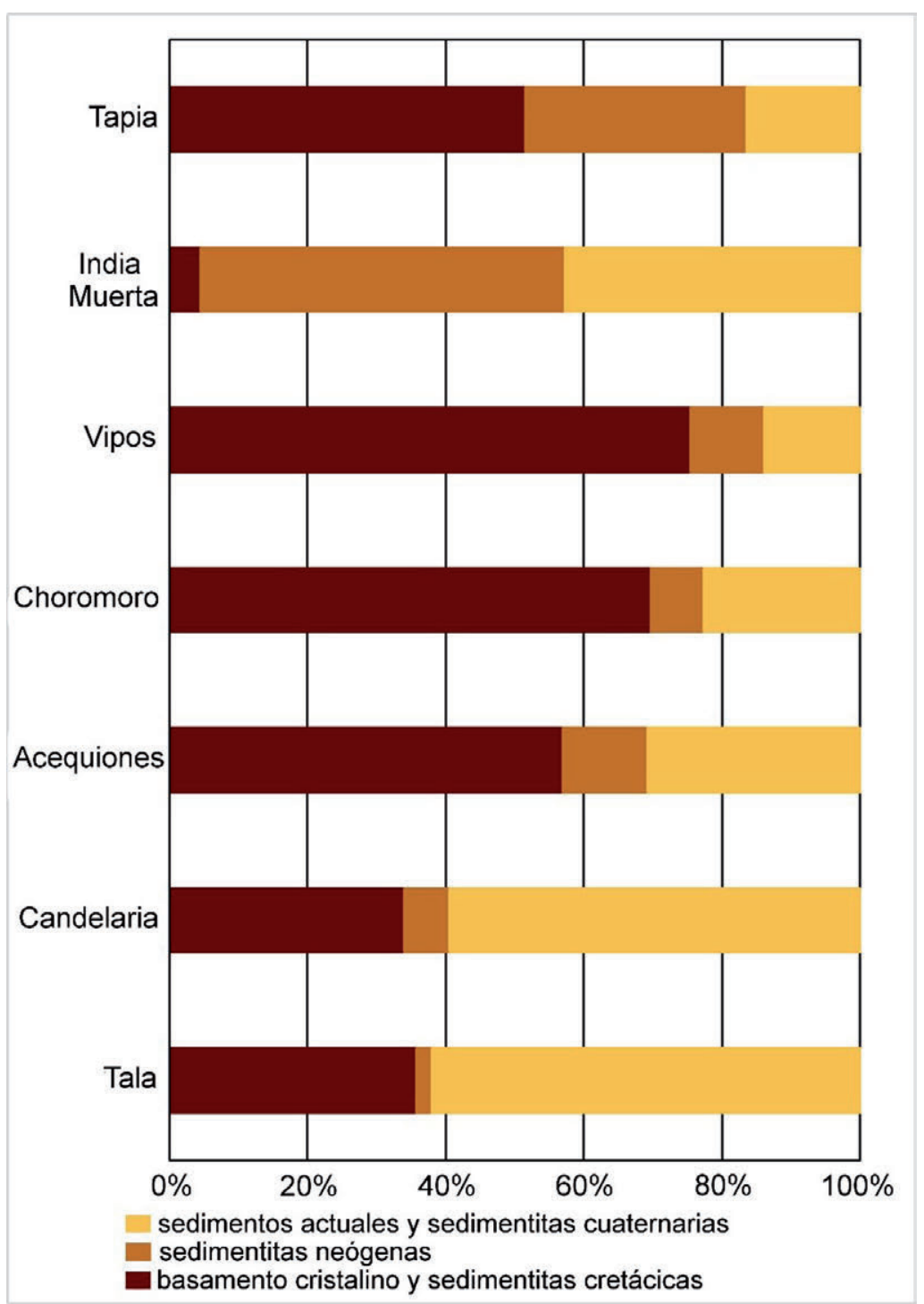

Figura 7. Proporción de las diferentes litologías en las subcuencas de los principales tributarios del Salí en la cuenca de Tapia-Trancas.

pendiente, proclives a presentar eventos de inundación (Campo et al. 2012); también se vinculan estos valores bajos a la heterogeneidad geológica, bajo control estructural y alta permeabilidad (Hajam et al. 2013). En este estudio únicamente la cuenca del río Candelaria presenta un valor que se encuentra en el rango de 3-5 indicativo de áreas de montaña con pendientes fuertes pero sin demasiado control estructural (Campo et al. 2012; Diaz Gómez et al. 2015). No se obtuvieron valores mayores a 5 que son reconocidos en la bibliografía como indicativos de control estructural en el desarrollo de la red de drenaje (Rai et al. 2017). Debe destacarse que las redes de drenaje resultantes del procesamiento automático en SIG no muestran todos los tributarios de orden 1 de las redes de drenaje; sin embargo, la metodología ha probado ser útil por el menor tiempo de procesamiento, en relación a métodos tradicionales y la capacidad gestionar diversos tipos de datos que facilitan la comprensión de los procesos que controlan el comportamiento de las redes de drenaje (Singh et al. 2017). 
Estos resultados pueden ser mejorados combinando los datos de teledetección con el análisis de información preexistente en mapas y fotografías aéreas.

El coeficiente de almacenamiento ( $R o$ ) indica el grado de desarrollo del drenaje en una cuenca y es controlado tanto por factores climáticos, geológicos, biológicos, y geomorfológicos como por causas de origen antropogénico (Horton 1945). Los valores altos obtenidos para los ríos Choromoro, Tapia y Acequiones implicarían que los caudales de punta se alcanzan en mayor tiempo y, por lo tanto, los procesos erosivos estarán atenuados. El caso contrario lo representan los ríos Candelaria y Tala que muestran los menores valores de coeficiente de almacenamiento, por lo que para estas cuencas se esperan procesos erosivos intensos y que los caudales máximos se alcancen en un corto tiempo (Biswas et al. 2014; Pande y Moharir 2017).

El coeficiente de torrencialidad $(\mathrm{Ct})$ permitió estimar la susceptibilidad a la erosión de las redes de drenaje estudiadas. Este índice considera la génesis erosiva de los ríos de orden 1 y los considera como los de mayor importancia en la generación de ûujos torrenciales y de inundaciones aluvionales veloces (Busnelli y Horta 2014). Este parámetro también indica la intensidad de los procesos de erosión lineal y la capacidad de descarga de una cuenca (Díaz Gómez et al. 2017). Los datos obtenidos muestran que la cuenca de menor susceptibilidad es la del río Choromoro, seguida de las cuencas de los ríos Vipos, Acequiones y Tapia con valores intermedios. Los valores más altos (mayor riesgo) caracterizaron a las cuencas de los ríos Candelaria,

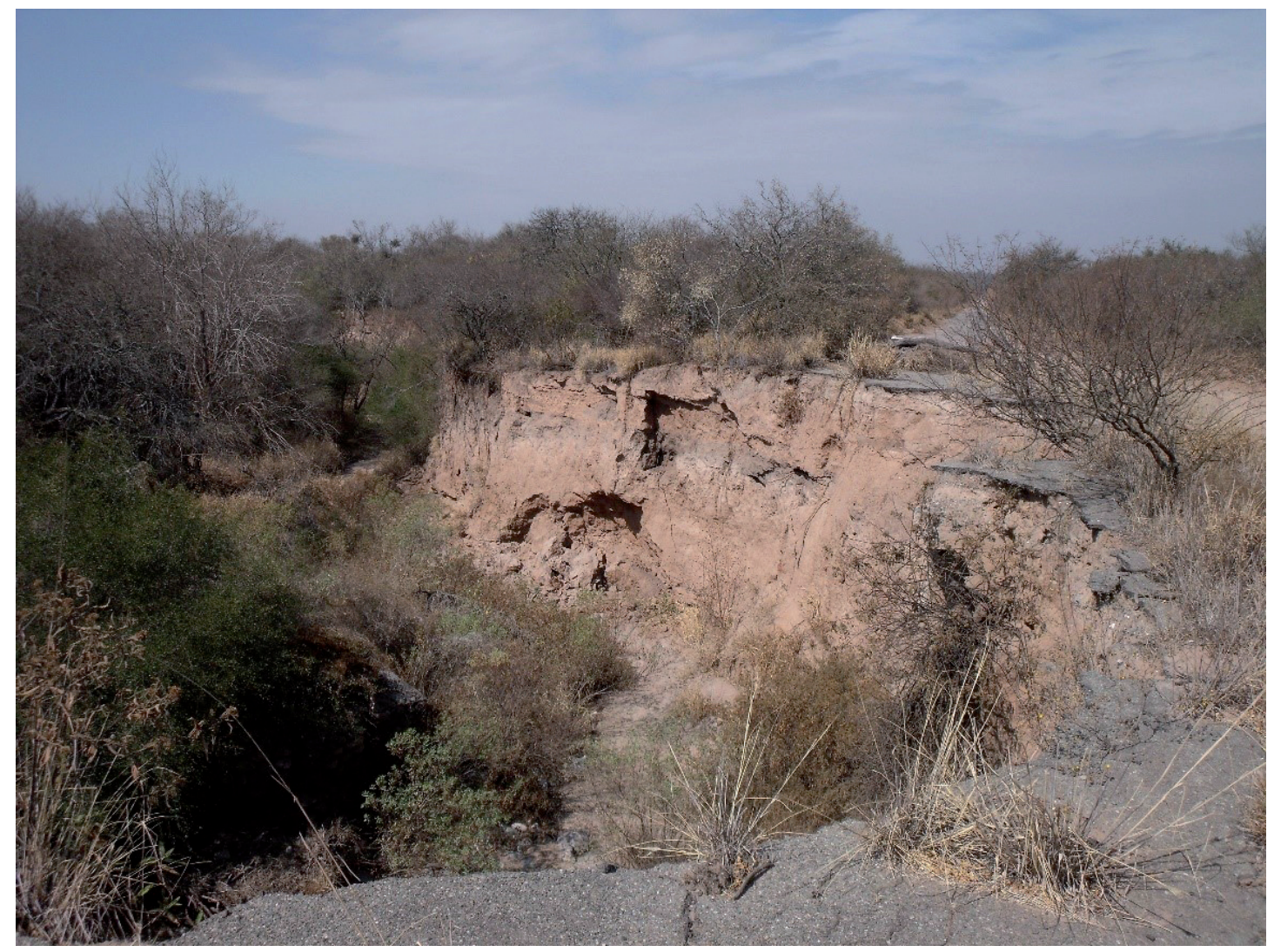

Figura 8. Procesos erosivos en el arroyo India Muerta, antigua traza de Ruta Nacional No 9 entre las localidades de Tapia y Vipos. 
Tala y arroyo India Muerta (Figura 8). Estos últimos, serían por lo tanto los de mayor susceptibilidad a la erosión, menores tiempos de llegada al pico de creciente y alta torrencialidad.

La extensión media del escurrimiento fue mínima para la subcuenca del arroyo India Muerta mientras que las redes de drenaje de los ríos Acequiones, Candelaria y Tala tuvieron valores intermedios y los ríos Tapia y Choromoro tuvieron los valores máximos. Estos resultados implicarían un mayor tiempo para alcanzar los caudales de punta en las cuencas de Tapia y Choromoro, mientras que los tiempos serían más cortos en las cuencas de India Muerta, Acequiones, Candelaria y Tala.

Valores altos de densidad de drenaje caracterizan redes de drenaje más finamente divididas, mientras que valores bajos de Dd indican menor textura (Gordon et al. 2004). Entre las subcuencas estudiadas, la del río Acequiones fue la de mayor densidad de drenaje seguida en orden descendente por los ríos Candelaria, Tala y Vipos, siendo el río Choromoro y el Arroyo India Muerta los de menor densidad. La densidad de drenaje se relaciona a las características climáticas, la geología y la cobertura vegetal de las cuencas. De acuerdo a Busnelli (2009) las redes hidrológicas desarrolladas sobre rocas impermeables presentan una mayor densidad de drenaje que aquellas desarrolladas sobre rocas permeables, bajo condiciones de clima similar. Las regiones semiáridas tienen mayores densidades de drenaje que las regiones húmedas debido a la escasa cobertura vegetal. Busnelli y Horta (2014) refieren que valores altos de densidad de drenaje indicarían el predominio del escurrimiento sobre la infiltración, bajo almacenamiento subsuperficial y subterráneo, rocas o sedimentos de texturas finas de relativamente baja permeabilidad y elevada susceptibilidad a la erosión. Kulkarni (2013) define que una alta densidad de drenaje indica que el material subsuperficial es impermeable, con vegetación dispersa y relieve alto. Mientras que una baja densidad de drenaje representa cuencas escasamente drenadas y respuesta hidrológica lenta.

La frecuencia de ríos (Fr) se relaciona con el comportamiento del escurrimiento superficial en las cuencas y valores altos indican mayor disponibilidad de agua en superficie, menor permeabilidad de los terrenos y mayor susceptibilidad a la erosión. La Fr también indica un diseño distintivo de la red de drenaje, condicionado por la estructura de las rocas, capacidad de infiltración, cobertura vegetal, naturaleza del relieve, cantidad de precipitación y permeabilidad del material subsuperficial. En este sentido, valores bajos de Fr estarían indicando la existencia de material subsuperficial permeable (Kulkarni, 2013). La Fr obtenida para el Arroyo India Muerta es indicativa de un escaso desarrollo, lo cual se comprueba por su baja densidad de drenaje y escasa superficie. Las subcuencas de los ríos Choromoro, Vipos, Acequiones, Candelaria, Tala y Tapia, siguieron en orden creciente de frecuencia de ríos, indicando una mayor proporción de ríos por unidad de área. Finalmente, los cambios observados en los perfiles de los ríos, relacionados a la presencia de quiebres de pendiente serían consecuencia de cambios en la geología del sustrato (OMM, 1994). 


\section{AGRADECIMIENTOS}

Los autores agradecen a los revisores anónimos del manuscrito por sus comentarios, que ayudaron a mejorar este trabajo. Los resultados presentados en este trabajo fueron financiados con una beca doctoral de CONICET.

\section{BIBLIOGRAFÍA}

Abascal, L. d. V. 2005. Deformación andina en la Cuenca de Choromoro, NO de Tucumán: estilo estructural combinado. Revista de la Asociación Geológica Argentina 60(2): 268-277.

Adler, F. J. 2004. Embalse El Cadillal (Prov. de Tucumán) situación actual y futura. CET Revista de Ciencias Exactas e Ingeniería Facultad de Ciencias Exactas y Tecnología U.N.T. 13(25): 48-56.

Beltramone, C. 2007. Geomorfología Fluvial del río San Antonio. Provincia de Córdoba. Argentina. Cuadernos del Curiham 13: 19-29.

Biswas, A., D. Das Majumdar y S. Banerjee. 2014. Morphometry Governs the Dynamics of a Drainage Basin: Analysis and Implications. Geography fournal. http:// dx.doi.org/10.1155/2014/927176.

Busnelli, J. 2009. Evolución Histórica, Situación Actual y Perspectivas Futuras del Riesgo de Inundación en la Cuenca del Río Gastona. Tucumán. Argentina. Tesis Doctoral inédita, Universidad Nacional de Tucumán, Tucumán.

Busnelli, J. y L. R. Horta. 2014. Morfometría de cuencas montanas y metamorfosis fluvial, Tucumán. Revista de la Asociación Geológica Argentina 71(1): 11-20.

Bustos, M. S. 2014. Las causas naturales y antrópicas que afectan los ríos y embalses actuales: identificación, jerarquización y cuantificación: Cuenca Tapia, Trancas, Tucumán, Argentina. Tesis doctoral, Universidad Nacional de Tucumán.

Bustos, M. S. y S. M. Georgieff. 2016. Erosión y producción de sedimento en una cuenca semiárida: causas y efectos en relación a la colmatación del embalse C. Gelsi. Presentado en el VIII Congreso Internacional de Control de la Erosión y Sedimentos. Cartagena, Colombia, 14-17 agosto de 2016. https://iecaiberoamerica.org/erosion-y-produccion-de-sedimento-en-una-cuenca-semiarida-causasy-efectos-en-relacion-a-la-colmatacion-del-embalse-c-gelsi/ (accedido el 11 de agosto de 2020).

Campo, A. M., N. B Aldalur y S. N. Fernández. 2012.Morfometría ûuvial aplicada a una cuenca urbana en Ingeniero White, República Argentina. Investigaciones Geográficas, Boletín del Instituto de Geografía UNAM 77: 7-17.

Córdoba, A. S. 2013. Análisis sismoestratigráfico y modelo tectosedimentario del límite Salta - Tucumán y Noroeste de Santiago del Estero. Seminario, Universidad Nacional de Tucumán.

Delgado, M. I. y F. J. Gaspari. 2010. Caracterización morfométrica geoespacial. Estudio de caso: Arroyo Belisario, Argentina. Tecnociencia Chihuaha 6(3): 154-163. 
Díaz Gómez, R., F. J. Gaspari y S. M. Georgieff. 2017. Aspectos morfométricos de cuencas subtropicales del Noroeste de Argentina. Acta geológica lilloana 29(1): 3-19.

Díaz, B. G., P. Tiberi y G. Marderwald. 2015. Morfometría de la Cuenca Alta del río Gallegos (Patagonia Austral, Argentina). Trabajo presentado en el XXV Congreso Nacional del Agua CONAGUA 2015, Paraná, 15 al 19 de junio de 2015.

Díaz, L. R. 2006. Sedimentación en embalses y su proyección como herramienta de gestión: análisis de un caso real. Presentado en el III Congreso Iberoamericano sobre control de la erosión y los sedimentos, Buenos Aires, 9 al 11 de agosto de 2006.

Doffo, N. y G. González Bonorino. 2005. Caracterización morfométrica de la cuenca alta del arroyo Las Lajas, Córdoba: Un análisis estadístico. Revista de la Asociación Geológica Argentina 60(1): 16-22.

Esper Angillieri, M. Y. y L. P. Perucca. 2014. Caracterización morfométrica de la Cuenca del río Seco a propósito de las fuertes precipitaciones de enero de 2013, Departamento Sarmiento, San Juan, Argentina. Boletín de la Sociedad Geológica Mexicana 66(2): 235-245.

Esper Angillieri, M. Y., K. Espejo, G. Lara, L. Perucca y M. Rothis. 2016. Morfometría de Cuencas como base en la evaluación de la susceptibilidad/peligrosidad por aluviones, Caso De Estudio: Cuenca del Río del Agua, San Juan, Argentina. Revista de la Asociación Geológica Argentina 73(4): 513-519.

Farr, T. G., P. A. Rosen, E. Caro, R. Crippen, R. Duren, S. Hensley, M. Kobrick, M. Paller, E. Rodriguez, L. Roth, D. Seal, S. Shaffer, J. Shimada, J. Umland, M. Werner, M. Oskin, D. Burbank y D. Alsdorf. 2007. The Shuttle Radar Topography Mission. Reviews of Geophysics, 45(2). doi: https://doi.org/10.1029/ 2005RG000183.

Fleming, M. J. y J. H. Dohan. 2009. HEC-GeoHMS Geospatial Hydrologic Modeling Extension. User's manual. Version 4.2. Davis: U.S. Army Corp of Engineers. Institute of Water Resources, Hydrologic Engineering Center.

Gaspari, F. J., A. M. Rodríguez Vagaría, G. E. Senisterra, G. Denegri, M. I. Delgado y S. Besteiro. 2012. Caracterización morfométrica de la cuenca alta del río Sauce Grande, Buenos Aires, Argentina AUGM DOMUS 4: 143-158.

Genchi, S. A., M. E. Carbone y G. M. E. Perillo. 2011. Hydrologic response of the drainage basins that intersect Las Grutas town, Argentina. Investigaciones Geográficas, Boletín del Instituto de Geografía UNAM 75: 23-36.

Georgieff, S. M. L. M. Ibañez, M. E. Vides, K. Anis, S. M. Nieva. 2014. Paleógeno y Neógeno de Tucumán: estratigrafía y paleoambientes sedimentarios. En Moyano, M. S., M. E. Puchulu, D. S. Fernández, M. E. Vides, S. Nieva, G. Aceñolaza Eds., Geología de Tucumán, 106-123, Colegio de Graduados en Ciencias Geológicas de Tucumán.

Gil, V. 2011. Geomorfología fluvial de la cuenca del arroyo El Negro, Buenos Aires, Argentina. Revista Universitaria de Geografía 20: 151-169.

Gil, V., J. O. Gentili y A. M. Campo. 2009. Influencia de la Litología en la Variación de los Parámetros Morfométricos, Sistema de Ventania, Argentina. Papeles de Geografía 49-50: 55-68. 
González, O. E., M. E. Viruel, R. Mon, P. Tchiliguirian y E. Barber. 2000. Hoja Geológica 2766-II San Miguel de Tucumán. Provincias de Tucumán, Catamarca, Salta y Santiago del Estero. Boletín, 245.

Gordon, N. D., T. A. McMahon, B. L. Finlayson, C. J. Gippel, R. J. Nathan. 2004. Stream ecology. An introduction for ecologists. 2nd edition. Chichester: J. Wiley and Sons, Ltd.

Guido, E. Y. 1987. Geomorfología básica y aplicada de la cuenca del río Tipas. Departamento Trancas - Provincia de Tucumán. Seminario, Universidad Nacional de Tucumán.

Hajam, R. A., A. Hamid y S. Bhat. 2013. Application of Morphometric Analysis for Geo-Hydrological Studies Using Geo-Spatial Technology -A Case Study of Vishav Drainage Basin. Hydrology: Current Research 4(3). doi: 10.4172/21577587.1000157.

Horton, R. E. 1945. Erosional development of streams and their drainage basins; hydrophysiscal approach to quantitative morphology. Bulletin of the Geological Society of America 56: 275-370.

Huespe, J., H. Suppo, D. Tabernig, S. Mangini, C. Romano, S. Schmidt, S. Oldani, S. Cañete, R. Mir, N. Fernández, M. Méndez y D. Cassini. 2009. Estudios de Batimetría 2009. Embalse El Cadillal. Tucumán: INCOCIV S.R.L.

Knighton, D. 1998. Fluvial forms and processes. A new perspective. London: Hodder Arnold.

Kulkarni, M. D. 2013. The Basic Concept to Study Morphometric Analysis of River Drainage Basin: A Review. International Fournal of Science and Research (IFSR), 4(7): 2277-2280.

Kumar, N. 2013. Morphometric Analysis of River Catchments Using Remote Sensing and GIS (A Case Study of the Sukri River, Rajasthan). International Fournal of Scientific and Research Publications 3(6). http://www.ijsrp.org/research-paper0613/ijsrp-p1823.pdf (accedido el 7 de marzo de 2018).

Mendoza, E. A. y J. A. González. 2011. Las ecorregiones del Noroeste Argentino basadas en la clasificación climática de Köppen. Serie Conservación de la Naturaleza 19.

Mesa, L. M. 2006. Morphometric Analysis of a Subtropical Andean Basin (Tucumán, Argentina. Environmental Geology 50(8): 1235-1242.

Nageswara, R. K., L. P. Swarna, K. P. Arun y K. M. Hari 2010. Morphometric Analysis of Gostani River Basin in Andhra Pradesh State, India Using Spatial Information Technology, International Fournal of Geomatics and Geosciences 1(2): 179-187.

Navarro, E. L. y D. M. Cano 2017. Análisis morfométrico comparativo de los tramos superiores de las cuencas de los arroyos Sauce Chico, Napostá y Sauce Grande, Sierras Australes: uso de técnicas digitales. Presentado en el IV Congreso Internacional Científico y Tecnológico de la Provincia de Buenos Aires. Quilmes, Buenos Aires, 1 de septiembre de 2017. https://igital.cic.gba.gob.ar/bitstream/ handle/11746/6712/11746_6712.pdf?sequence $=1 \&$ isAllowed $=\mathrm{y}$ (accedido el 02 de mayo de 2018). 
Organización Meteorológica Mundial. 1994. Guía de prácticas hidrológicas: adquisición $y$ proceso de datos, análisis, predicción y otras aplicaciones. Quinta edición, $\mathrm{OMM}-\mathrm{N}^{\mathrm{O}}$ 168. Ginebra: Organización Meteorológica Mundial.

Pacha Consultora Ambiental S.R.L. 2005. Batimetría y estudio de colmatación del embalse de El Cadillal. Tucumán: Pacha Consultora Ambiental.

Pande, C. B. y K. Moharir 2017. GIS based quantitative morphometric analysis and its consequences: a case study from Shanur River Basin, Maharashtra India. Applied Water Science 7: 861-871.

Rai, P. K., K. Mohan, K. M. S. Mishra y A. Ahmad. 2017. A GIS-based approach in drainage morphometric analysis of Kanhar River Basin, India. Applied Water Science 7(1): 217-232.

Renschler, C. S., C. Mannaerts y B. Diekkrüger. 1999. Evaluating spatial and temporal variability in soil erosion risk-rainfall erosivity and soil loss ratios in Andalusia, Spain. Catena 34: 209-225.

Salomón, M. y D. Soria 2002. Estudio Hidrográfico e Hidrológico de las Cuencas del Río Seco Chacras de Coria y Tejo. Presentado en XIX Congreso Nacional del Agua. Villa Carlos Paz, Córdoba, Argentina, agosto 2002. http://asicprimerazona.com.ar/asic/publicaciones/estudio_tejo_mendoza.pdf (accedido el 2 de mayo de 2018).

Singh, P., A. Gupta, y M. Singh. 2014. Hydrological inferences from watershed analysis for water resource management using remote sensing and GIS techniques. The Egyptian Fournal of Remote Sensing and Space Sciences, 17: 111-121.

Strahler, A. N. 1957. Quantitative Analysis of watershed geomorphology. Transactions of the American Geophysical Union 38(6): 913-920.

Torrella, S. A. y J. Adámoli. 2005. Situación Ambiental de la Ecorregión del Chaco Seco. En Brown A., Martínez Ortiz, U., Acerbi, M. y Corcuera, J. eds. La Situación Ambiental Argentina 2005, 73-100. Buenos Aires: Fundación Vida Silvestre. http://www.fvsa.org.ar/situacionambiental/chacoseco.pdf (accedido el 2 de mayo de 2018). 